ISSN 1516-635X Apr - Jun 2010/v.12/n.2/115 - 119

Workshop: Infectious Bronchitis (IB) in the Brazilian Poultry Industry

\section{Etiology and Immunology of Infectious Bronchitis Virus}

\section{ABSTRACT}

Infectious bronchitis virus (IBV) of chickens is currently one of the main diseases associated with respiratory syndrome in domestic poultry, as well as with losses related to egg production. The etiological agent is a coronavirus, which presents structural differences in the field, mainly in the S1 spike protein. The immune response against this virus is complicated by the few similarities among serotypes. Environmental and management factors, as well as the high mutation rate of the virus, render it difficult to control the disease and compromise the efficacy of the available vaccines. Bird immune system capacity to respond to challenges depend on the integrity of the mucosae, as an innate compartment, and on the generation of humoral and cell-mediated adaptive responses, and may affect the health status of breeding stocks in the medium run. Vaccination of day-old chicks in the hatchery on aims at eliciting immune responses, particularly cell-mediated responses that are essential when birds are first challenged. Humoral response ( $\lg Y$ and $\lg A$ ) are also important for virus clearance in subsequent challenges. The presence of antibodies against the $\mathrm{S} 1$ spike protein in 3- to 4-week-old birds is important both in broilers and for immunological memory in layers and breeders.

\section{INTRODUCTION}

Commercial poultry production is very sensitive to results, and therefore, there is very little room for long-term management strategies. As expected in any animal production industry, better results in poultry production obviously require constant adaptation and novel strategies. Such high sensitivity may improve results and speed up growth and modernization; however, management errors otherwise small, may compromise potential live performance.

Health management has constantly developed to ensure the health of flocks at the best cost-benefit ratio, which is achieved when factors involved in diseases are well understood. The right information must be available at the time it is needed. This is particularly important when chicken infectious bronchitis (IB) is considered, as it is highly prevalent in Brazil and its control measures still require improvement.

\section{ETIOLOGY}

The Infectious Bronchitis Virus (IBV) is a coronavirus. It was first isolated in 1930. By definition, IBV is a coronavirus that affects domestic chickens, and replicates in respiratory tissues, as well as in other epithelial tissues, including the kidneys, gonads, and bursa (Cavanagh, 2007a). However, virologists have found it difficult to precisely determine in which types of tissue these viruses replicate and even in which animal species, as it was observed that it may overcome hosts' barriers, as in the case of 
SARS (severe acute respiratory syndrome) (Cavanagh, 2005; Enjuanes et al., 2006). The known coronaviruses are classified in three groups, based on their antigenic differences and confirmed by genetic sequencing. IBV is classified in group 3, and its viral proteins share less than $40 \%$ amino-acid identity with the other viruses in the group. Group 1 is more heterogeneous, and was subdivided into two subgroups - $1 \mathrm{a}$ and $2 \mathrm{a}$ - which share less than $45 \%$ identity. By the end of 2002 , the occurrence of SARS in China allowed the characterization of a previously unknown group. The proteins of this new virus have little identity with other already described coronaviruses, and therefore the creation of group 4 was proposed for its classification (Cavanagh, 2003), although recent genetic studies suggest that this new virus derives from group 2 viruses, and therefore should be classified in group $2 \mathrm{~b}$ (McKinley, et al., 2008). Recently, the Coronavirus Study Group of the International Committee for Taxonomy of Viruses (ICTV) proposed three genera: Alpha, Beta, and Gamma coronaviruses, subdivided into subgroups 2a, 2b, 2c, and 2d; and 3a, 3b, and 3c, replacing the traditional groups 1,2 , and 3 (Woo, 2009).

These genera consist of enveloped viruses with a single-stranded RNA with positive-sense genetic material. They present a large surface protein - $\mathrm{S}$ or spike protein -, responsible for the adsorption of the virus into the cell and for the fusion of the viral envelope with the endosomal or cell membrane for subsequent release of virus RNA in the cytoplasm. There are three other structural proteins (N, E, M). Group 2 also presents a membrane protein (hemagglutinin-esterase, $\mathrm{HE}$ ), and some groups of virus express protein I. The main membrane protein of SBVs is the S protein, or spike protein, which, due its position in the viral envelope, determines the "crown" shape of these viruses as seen under electron microscopy, which accounts for the denomination of the coronaviridae family. The $S$ protein occurs as a dimer or a trimer (Lewicki \& Gallagher, 2002), and can be cleaved into two subunits, S1 (amino-terminal component) and S2 (carboxy-terminal component). This spike protein is important to determine virus specificity and it is also involved in the pathogenicity of the virus (Zeng, et al., 2006).

IBV serotype classification is based on S1 spike protein differences. Many serotypes present 20 to $25 \%$ differences in $\mathrm{S} 1$ amino acids; others, more than $50 \%$. Differences in other viral proteins rarely exceed $15 \%$. Thus, generally speaking, immunity against one serotype provides poor protection against other serotypes, considering that cross-protection decreases when differences are higher than 5\%. Experimentally, virus recombination rate, specifically in the $\mathrm{S}$ spike protein, can reach $50 \%$, evidence that supports the hypothesis of virus evolution in the field. (Cavanagh, et al., 2007-b).

The virus initially infects the upper respiratory tract, and three days after inoculation of virus replication, the highest titers are found in the trachea (Cavanagh, 2003), which may persist up to five days depending on the virus strain. IBV encodes proteins that modulate and induce apoptosis, sometimes directly contributing to virus pathogenicity, or inhibit apoptosis, as the prevention of the early death of infected cells allows viral replication and generation of high titers in the infected animal (Enjuanes, et al., 2006).

Such balance of pro- or anti-apoptotic molecules affects cell survival in the early stages of infection. Interestingly, pro-apoptotic molecules target specific tissues, influencing the clinical manifestations of the disease, whereas anti-apoptotic molecules maintain the virus population longer, allowing virus mutation and adaptation. This leads to the emergence of virus variants for three main reasons: RNA-polymerase infidelity, the possibility of recombination among the strains, and a larger RNA genome with assimilation of genomic changes.

\section{IMMUNOLOGY}

Due to the great versatility of coronaviruses in infecting different tissues, T- lymphocyte CD4 and CD8 populations may be affected in some infection models, interfering with virus clearance since it impairs birds adaptive responses. Both humoral and cell-mediated responses are important in the control of infections, and currently any field challenge that causes immunosuppression predisposes birds to infectious bronchitis or other respiratory syndromes. Innate immunity is crucial for the control of IBV field infections, and its early activation is based mainly on the action of Interferon-gamma, as a result of the action of macrophages, in addition to other substances, during early inflammatory process (Catani et al., 2000).

A large number of innate factors are involved in the early recognition of the virus, such as TLR-3 (tool-like receptor) in the macrophages (Arians et al., 2008). Similarly, other cytokines have been recognized in response to different respiratory viral infections caused by RNA viruses. These cytokines are synergistically involved in the detection of the presence of the virus, triggering a cascade of other factors, which result not 
only in the innate protection of adjacent cells, but also facilitate the activation and migration of T-lymphocytes to the challenge sites, determining adaptive responses (Goo et al., 2008). These adaptive responses of the birds against the virus are the basis of the development of humoral and cell-mediated immune responses. These responses are essential, as the presence of high titers of systemic antibodies is correlated with the failure in detecting viruses in the kidneys and in the argental tract, as well as with the absence of decreased egg production or quality (Mondale \& Nazi, 2001).

The presence of $\lg A$ in the respiratory mucosa is associated with resistance to infections, confirming its role in the inhibition of the viral infection at its preferred site. As the chicken can produce up to $100 \mathrm{mg} / \mathrm{kg} /$ day of $\lg A$, as opposed to only $30 \mathrm{mg} / \mathrm{kg} /$ day of $\lg Y$, the need and importance of such mucosal response may be inferred. Despite being induced by live vaccines, this response is not measured in health monitoring. Although the immune response against IBV is very complex, bird susceptibility is influenced by their MHC (Major Histocompatibility Complex) genotype. MHC determines the quality of humoral and cell-mediated responses, as the MHC molecule is responsible for binding the antigen epitope and to present it to Tlymphocytes (Joiner et al., 2007). The presentation of the main epitopes, including the S1 spike protein, by specialized antigen-presenting cells is the starting point of the generation of humoral (when the epitope binds to MHC-II) or cell-mediated (when the epitope binds to $\mathrm{MHC}-\mathrm{I}$ ) responses.

During natural infection, immune responses are balanced, but, after vaccination, factors such as vaccine strain, bird age, and MHC-I and MHC-II expression, as well as CD4 to CD8 ratio, may vary, exerting higher selection pressure on the virus. As with other RNA viruses, the immune response induced by infection, and especially by the use of vaccines, exerts a selection pressure that allows the virus to adapt the host and to the environment over time due to its ability to mutate (Zinkernagel, 2003). The absence of a proof-reading mechanism in virus replication is one of the predisposing and determining factors of the observed mutation rate of about 10-3, which is similar to the influenza virus mutation. IBV mutation rate was established as 1.5\% $-2.5 \%$ per year in the presence of vaccination, and as $0.3 \%$ without vaccination. In passages in cell cultures, a change of 25 amino acids in S1 after 7 passages is observed (McKinley et al., 2008). The development of future vaccines against infectious bronchitis must take into account studies that evaluated the neutralizing capacity of antibodies anti-S2 protein as, despite being less effective as compared to $\mathbf{S 1}$, the preserved genome of the S2 protein may be important (Zeng et al., 2006).

In this context, the correct use of tools to IBV infection must be evaluated and all factors must be considered, from providing environmental good conditions (including bird density, single-age flocks, biosecurity, etc.) up to understanding how and when stimulating the immune system to provide protection agains the disease.

Bird capacity to respond to a health challenge at any age depends on the proper development of the immune system, from the embryonic life up to maturity, around 3 to 6 weeks of age. The presence of antibodies or cytotoxic T-cells reacting specifically to the antigen depends on the quality of the bird's bursa and thymus up to sexual maturity. Any factor that affects these organs during their development will result in higher metabolic cost due to the immune response cascade, consequently affecting the remaining of the bird's life (van Ginkel et al., 2008). On the sixth day of embryonic life, the thymus begins to be colonized by undifferentiated cells that will become T-lymphocytes, reaching a peak in 15 or 16 days. After the 10th day of embryonic life, the bursa starts to be colonized. At hatch, these organs are anatomically developed, but functional maturation is accelerated by external stimuli. As environmental challenges are very different, the presence of maternal antibodies in newly-hatched chicks ensures a response to the external challenges present during the early stages of life. The importance of that passive protection is different according to the disease, but $\lg G(\lg Y)$ antibodies, transmitted from the hen to the chick via yolk, can usually be detected in the serum and in respiratory mucosa of chicks.

Different studies have shown that maternal antibodies may control challenges for an initial period that varies from 7 to 14 days, depending on the antibody determination method, challenge type, and virus studied (Mondal \& Naqi, 2001). The control of viral infections in the body entry sites is highly influenced by the local adaptive and innate response after immunization (Guo et al., 2008). The classic role of the mucosal vaccination, which consists in stimulating $\lg A$ production, is still controversial when the infectious bronchitis model is studied. Many primary and secondary post-infection tests, including the characterization of the memory against IBV, have shown high levels of local IgY. Pei \& Colisson (2005) demonstrated that the presence of $\lg A$ is important in 
controlling the infection and those long-life plasma cells, as those found in mammal bone marrow, are observed in the spleen of birds. IgY levels may be present for at least 18 weeks, ensuring rapid reaction when a challenge emerges. Pei et al. (2003) point out that vaccinating 1-day-old chicks may be ineffective, but may be an important time for a first controlled vaccineinduced challenge.

Similarly, the action of local cell-mediated response in prime infection seems to clearly accelerate viral clearance, but its importance is minimal at a second exposure, because the neutralization of the pathogen by IgY is immediate (Guo et al., 2008). Cytotoxic Tcells are detected 3 days after infection by IBV and peak in 10 days. During that period, infection is normally resolved and the virus is eliminated (Pei \& Colisson, 2003). After that, the number of CTLs (cytotoxic Tlymphocytes) drops, together with virus titers in the respiratory system and in the kidneys. Two weeks after the infection, the number of cells is not sufficient to protect the bird. This may be explained by the high metabolic cost of that response, which is reduced since it is no longer necessary. The current vaccination protocols aim mainly at taking advantage of the management situation, as the day-old chicks leave the hatchery already vaccinated, and of the fact that, at placement, chicks are protected by $\lg A$ or $\lg Y$, in addition to cell-mediated response. The response capacity to vaccination stimulus is already present at hatch, but it is different from that of a two- to threeweek-old bird, for instance, due to the maturation of the immune organs.

The presence of an attenuated vaccine virus in the respiratory system is intended to prevent its colonization by wild viruses. This is called immune exclusion and, as mentioned above in the case of IBV, there may be undesirable consequences over time, not only due to the selection pressure, but also by reversion and mutation of the vaccine virus, which may become uncontrollable variable in the medium run. The question as to how long this active protection lasts after a dose of live vaccine at one day of age does not have a simple answer, because it involves challenge intensity, flock uniformity, presence of maternal antibodies, homology of the vaccine strain, etc. There is little doubt as to the importance of innate immunity (local and systemic) and of cell-mediated immunity in virus clearance after primary infection. Thus, it must be noted that, among immune mechanisms in poultry, constitutive innate immunity as the first line of defense, when the presence and integrity of the affected cells and tissues may ensure a rapid response at low metabolic cost. This type of response has low development, maintenance, and use costs, and causes birds to take longer to reach their performance genetic potential.

Induced innate response is observed when, after a challenge, cells are mobilized and various immune response mediators appear. Although this response has low development and maintenance costs, the cost of its utilization is high, as it promotes anorexia and depression. This may only after vaccination, but also after challenge with a wild virus. This is necessary to promote vaccination response, with is adaptive and specific, and determines immunological memory. Response specificity can be achieved in different ways and at different sites, according to the type of vaccine applied. As previously discussed, the aim is to obtain both humoral and cell-mediated responses in the mucosa after challenging it with replicating (live) vaccines. This type of vaccine also ensures systemic immunity, different from inactivated or non-replicating vaccines, which are used parenterally and determine a strong systemic response - mainly humoral - but not a mucosal response or even a cytotoxic T-lymphocytes (CD8) response (Talebi et al., 2005).

Neutralizing antibodies induced during infectious bronchitis are directed against the $\$ 1$ protein of the surface spike. After infection (p.i.), IgM peaks in 12 days, and falls up to day 20 p.i. The presence of $\lg Y$ is not normally detected until day 10 p.i., but it increases exponentially between days 20 and 30 p.i. The induction of cytotoxic T-lymphocytes by CD8 T-lymphocytes is very important in the early stages of infection. Those cells are detected for a long period at decreasing levels, as a result of a decrease in challenge (Colisson et al., 2000). The protective immunity against infectious bronchitis is complex and does not result only from the bird's sensitization with the specific spike protein or from interactions of specific antibodies in a future challenge. In addition to individual bird characteristics, which determine its response capacity, other proteins that are not important epitopes, but that may undergo combinations, thereby determining different pathogenicity, influence the bird's response capacity (Cavanagh, 2003).

\section{REFERENCES}

Ariaans MP, Matthijs MGR, Van Harlen D, Van de Haar P, Ivan Eck $J H H$, Hensen EJ, Vervelde, L. The role of phagocytic cells in enhanced susceptibility of broilers to colibacillosis after infectious bronchitis 
virus infection. Veterinary Immunology and Immunopathology 2008; (123):240-250.

Cavanagh D. Severe acute respiratory syndrome vaccine development: Experiences of vaccination against avian infectious bronchitis coronavirus. Avian Pathology 2003; 32(6):567-582.

Cavanagh D. Coronaviruses in poultry and other birds. Avian Pathology 2005; 34(6):439-448.

Cavanagh D. Coronavirus avian infectious bronchitis virus. Veterinary Research 2007; 38:281-297.

Cavanagh D, Casais R, Armesto M, Hodgson T, Izadkhasti S, Davies M, Lin F, Tarpey I, Britton P. Manipulation of the infectious bronchitis coronavirus genome for vaccine development and analysis of accessory proteins. Vaccine 2007; 25:5558-5562.

Colisson EW, Pei J, Dzielawa J, Seo SH. Cytotoxic T. Lymphocytes are critical in the control of infectious bronchitis virus in poultry. Developmental and comparative immunology. 2000; 24:187-200.

Enjuanes L, Almazán F, Sola I, Zuñiga S. Biochemical aspects of coronavirus replication and virus-host interaction. Annual Review Microbiology 2006; 60:211-230.

Guo X, Rosa AJM, Chen DG, Wang X. Molecular mechanisms of primary and secondary mucosal immunity using avian infectious bronchitis virus as a model system. Veterinary Immunology and Immunopathology 2008; 121:332-343.

Joiner KS, Hoerr FJ, Ewald SJ, van Santen VL, Wright JC, van Ginkel $\mathrm{FW}$, Toro H. Pathogenesis of infectious bronchitis virus in vaccinated chickens of two major histocompatibility B complex genotypes. Avian Diseases 2007; 51:758-763.

Kotani T, Shiraishi Y, Tsukamoto Y, Kuwamura M, Yamae J, Sakuma $S$, Gohda M. Ephithelial cell kinetics in the inflammatory process of chicken trachea infected with infectious bronchitis virus. Journal Veterinary Medicine Science 2000; 62(2):129-134.

Lewicki DN, Gallagher TM. Quaternary structure of coronavirus spikes in complex with carcinoembryonic antigen-related cell adhesion molecule cellular receptors. The Journal of Biological Chemistry 2002; 277(22):19727-19734.

McKinley ET, Hilt DA, Jackwood MW. Avian coronavirus infectious bronchitis attenuated live vaccines undergo selection of subpopulations and mutations following vaccination. Vaccine 2008; 26:1274-1284

Mondal SP, Naqi SA. Maternal antibody to infectious bronchitis virus: its role in protection against infection and development of active immunity to vaccine. Veterinary Immunology and Immunopathology 2001; 79:31-40.

Pei J, Briles E, Colisson EW. Memory T cells protect chicks from acute infectious bronchitis virus infection. Virology 2003; 306:376384.

Pei J, Colisson EW. Specific antibody secreting cells from chickens can be detected by three days and memory B cells by three weeks post-infection with the avian respiratory coronavirus. Developmental and Comparative Immunology 2005; (29):153-160.

Talebi A, Pourbakhsh SA, Dorostkar K. Effects of vaccination routes against IB on performance on immune response of broiler chickens. International Journal of Poultry Science 2005; 4(10):795-798.

Van Ginkel FW, Van Santen SL, Gulley SL, Toro H. Infectious bronchitis virus in the chicken harderian gland and lachrimal fluid: viral load, infectivity, immune cell responses, and effects of viral immunodeficiency. Avian Diseases 2008; (52):608-617.

Woo PCY, Lau SKP, Huang Y, Yuen KY. Coronavirus diversity phylogeny and interspecies jumping. Experimental Biology and Medicine 2009; 234:1117-1127.

Zeng F, Hon CC, Yip CW, Law KM, Yeung YS, Chan KH, Peiris JSM, Leung FCC. Quantitative comparison of the efficiency of antibodies against S1and S2 subunit of SARS coronavirus spike protein in virus neutralization and blocking of receptor binding: implications for the functional roles of S2 subunit. Federation of European Biochemical Societies Letters 2006; 580:5612-5620.

Zinkernagel RM. On natural and artificial vaccinations. Annual Review of Immunology 2003; 21:516-546. 